\title{
Dosimetric comparison between three- and four-dimensional computerised tomography radiotherapy for breast cancer
}

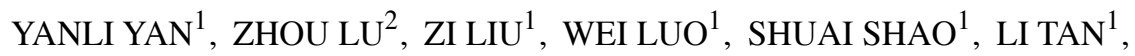 \\ XIAOWEI MA ${ }^{1}$, JIAXIN LIU ${ }^{1}$, EMMANUEL KWATENG DROKOW ${ }^{1}$ and JUAN REN ${ }^{1}$ \\ ${ }^{1}$ Department of Radiotherapy, Oncology Department, The First Affiliated Hospital of Xi'an Jiaotong University, \\ Xi'an, Shaanxi 710061; ${ }^{2}$ Department of Radiotherapy, Oncology Department, \\ Xi'an Gaoxin Hospital, Xi'an, Shaanxi 710075, P.R. China
}

Received April 17, 2018; Accepted April 12, 2019

DOI: $10.3892 / \mathrm{ol} .2019 .10467$

\begin{abstract}
At present, methods of radiotherapy simulation for breast cancer based on four-dimensional computerised tomography (4D-CT) or three-dimensional CT (3D-CT) simulation remain controversial. In the present study, 7 patients with residual breast tissue received whole breast radiotherapy based on 3D-CT and 4D-CT simulation. For the 4D-CT plan, four types of CT images were produced, including images of the end of inspiration and the end of expiration, and images acquired by the maximal intensity projection (MIP) and average intensity projection (AIP). In the 3D-CT plan, the clinical target volume (CTV) and plan target volume (PTV) were marginally higher compared with the 4D-CT plan. In addition, the minimum point dose of the target volume $\left(D_{\min }\right)$, the maximum point dose of the target volume $\left(D_{\max }\right)$ and the mean point dose of the target volume $\left(D_{\text {mean }}\right)$ of the CTV and PTV in the MIP and AIP plans were marginally higher compared with the 3D-CT plan. For the contralateral breast (C-B), volumes of the 4D-CT plan were markedly lower compared with the 3D-CT plan. Furthermore, $D_{\min }, D_{\max }$ and $D_{\text {mean }}$ of the 3D-CT plan were higher compared with the AIP and MIP plans. For the ipsilateral lungs (I-L), volumes of the 3D-CT and AIP plans were higher compared with the MIP plan. Furthermore, when breast lesions were on the left side, for the heart, the volume receiving no less than $40 \%$ of the prescription dose $\left(V_{40}\right)$ and the volume receiving no less than $30 \%$ of the prescription dose $\left(V_{30}\right)$ of the MIP and AIP plans were slightly lower compared with those of the $3 \mathrm{D}$ plan. In
\end{abstract}

Correspondence to: Professor Juan Ren, Department of Radiotherapy, Oncology Department, The First Affiliated Hospital of Xi'an Jiaotong University, 227 Yanta West Road, Xi'an, Shaanxi 710061, P.R. China

E-mail: 869491533@qq.com

Key words: three-dimensional computerised tomography, four-dimensional computerised tomography, maximal intensity projection, average intensity projection, breast cancer, radiotherapy, dosimetry conclusion, 4D-CT radiotherapy based on the MIP and AIP plans provides a slightly smaller radiation area and slightly higher radiotherapy dosage of the CTV and PTV compared with 3D-CT radiotherapy for breast radiotherapy. Therefore, the MIP and AIP plans prevent $\mathrm{C}-\mathrm{B}$ radiation exposure and improve sparing of the heart and I-L.

\section{Introduction}

The extensive use of whole breast radiotherapy has rendered it a standard treatment for patients with early breast cancer undergoing breast-conserving surgery (1-4). Previous advancements of individualised treatment for breast cancer have facilitated the gradual application of whole breast radiotherapy to neoadjuvant radiotherapy $(5,6)$ and palliative radiotherapy $(7,8)$, which have enhanced opportunities for surgical intervention, and improved the survival time and quality of life of patients with breast cancer.

At present, radiotherapy techniques for breast cancer are typically based on three-dimensional computerised tomography (3D-CT) simulation. However, the 3D-CT plan overlooks the target volume and radiotherapy dosage bias induced by breathing movements. Reportedly, 3D-CT images depend on different respiration phases when the CT scan starts (9). Previous studies have established that the accuracy and efficiency of radiotherapy in the chest and abdomen can easily be reduced by motions of tumours and organs at risk (OAR) $(10,11)$. To overcome these problems, a novel technological innovation, four-dimensional (4D)-CT radiotherapy technique, has emerged. Compared with conventional scanning, more accurate images of tumours and normal organs can be acquired by the 4D-CT process (11). At present, 4D-CT radiotherapy has been extensively applied in the treatment of lung cancer, liver cancer, oesophageal cancer, gastric cancer and kidney cancer; however, it has rarely been applied for breast cancer (11-19). For 4D-CT simulation, a breathing cycle is evenly divided into ten respiration phases and ten sets of CT images are acquired, respectively $(10,11)$. Typically, the outline of targets and OAR on all ten sets of images is delineated by radiation oncologists, which increases the workload compared with 3D-CT radiotherapy $(17,20,21)$. A number of composite methods have been used to decrease and optimise the work, 
including maximal intensity projection (MIP), average intensity projection (AIP), minimum intensity projection and two-extreme-phases fusion (21,22). While MIP and MIP-CT images are acquired by finding the maximum and minimum CT value along the slices at the same pixel location, AIP-CT images are acquired by averaging all CT values along the slices at the same pixel location $(22,23)$. The two-extreme-phases fusion method involves delineating target outlines on CT images of the two extreme respiration phases (T00 for the end of inhalation and T50 for the end of exhalation) and then fusing them (21). Notably, MIP-CT images are effective for assessing the motion of the organ but not for determining the tumour boundary near the diaphragm and chest wall, which can be readily demonstrated by AIP-CT and MIP-CT images (22). For partial breast irradiation, previous studies have demonstrated that 4D-CT radiotherapy improves the target definition and decreases the radiation dose of $\operatorname{OAR}(18,19)$. Furthermore, certain previous studies have compared different composite methods for lung cancer and liver cancer (21,23-28); however, to the best of our knowledge, at present, there is no study that has been published in English for breast cancer.

Therefore, the present study aimed to compare the target volumes and the dosimetric difference between the 3D-CT and 4D-CT plans for whole breast radiotherapy to determine the more effective radiotherapy technique for breast cancer.

\section{Patients and methods}

Patients and design. In the present study, seven female patients with breast cancer with residual breast tissue received whole breast radiotherapy based on 3D-CT and 4D-CT between March 2016 and April 2017 at The First Affiliated Hospital of Xi'an Jiaotong University (Xi'an, China). Inclusion criteria: i) Female patients were digonsed with breast cancer by pathology and and clinical examination; ii) patients were willing to accept and could tolerate breast radiotherapy; and iii) patients had no radiotherapeutic contraindication. Exclusion criteria: i) patients with metastatic or recurrent breast cancer; and ii) patients who had previously received chest radiotherapy. Table I summarises the characteristics of all patients. The present study was approved by The Ethics Committee of First Affiliated Hospital of Xi'an Jiaotong University (approval no. 2015-101). Written informed consent was obtained from all participants.

CT simulation. All seven patients were fixed in position using thermoplastic sheets or breast brackets. Subsequently, 3D-CT or 4D-CT scans were received sequentially with free breathing in the supine position. For each patient, images were obtained using a Philips Big Bore CT-Simulator (Philips Medical Systems, Inc., Bothell, WA, USA) with 5-mm slice thickness and a scan range from the submentum to the subphrenic, including the heart, bilateral breasts and bilateral lungs. All CT images were then uploaded and reconstructed on the Monaco 5.11.01 radiation treatment planning system (TPS) (ELEKTA Co., Sweden).

In addition, the 4D-CT scan was acquired using the Cine model and supplemented by the real-time position management (RPM) system (Philips Co., Holland) during breathing. Notably, the scan time was $>1$ respiratory cycle. For each patient, the respiratory cycle was evenly divided into ten respiration phases by the RPM system. T00 was defined as the end
Table I. Characteristics of the patients $(n=7)$.

\begin{tabular}{lll}
\hline Characteristic & Median (range) & $\mathrm{n}$ \\
\hline Age, years & $50.57(39-76)$ & \\
Disease stage & \\
Ia & \\
IIa & 2 \\
IIb & 3 \\
IV & 1 \\
Radiotherapy & 1
\end{tabular}

Radiotherapy location

Right breast 4

Left breast 3

Type of radiotherapy

Postoperative 4

Neoadjuvant 2

Palliative 1

Surgical treatment

Yes 5

No 2

Plan target volume, $\mathrm{cm}^{3} \quad 757.702(500.37-1,063.08)$

of inhalation and T50 was defined as the end of exhalation. Furthermore, ten sets of 4D-CT images of the ten respiration phases were acquired, and the MIP-CT and AIP-CT images of each patient were fused and reconstructed.

Targets and OAR delineation, and dose prescription. All acquired CT images were uploaded and rebuilt on the Monaco 5.11.01 TPS. Clinical doctors delineated the outlines of target areas and OAR, and medical physicists formulated radiotherapy plans. In addition, all delineations and the five types of plans (3D, T00, T50, MIP and AIP) were separately implemented for each patient by the same skilled doctor and medical physicist.

Target delineation. The clinical target volume (CTV) consisted of the whole residual breast tissue. The upper and lower boundaries of the CTV indicated the edges of breast tissue, the inner boundary indicated the sternal line, and the outer boundary indicated the anterior axillary line. The anterior boundary was $5 \mathrm{~mm}$ below the skin surface and the posterior boundary was the ectopectoralis fascia. In addition, the plan target volume (PTV) was attained by adding 5-mm isotropic expansion of the CTV and the anterior boundary was refined $3 \mathrm{~mm}$ below the skin surface simultaneously.

$O A R$ delineation. In the present study, the delineation method of the contralateral breast $(\mathrm{C}-\mathrm{B})$ was similar to the aformentioned method of the CTV. The heart was delineated from the right atrium and the right ventricle to the cardiac apex, excluding the pulmonary trunk, ascending aorta and vena cava. In addition, the right and left lungs were delineated by the automatic function of the Monaco TPS and manual modification. Furthermore, the spinal cord and bilateral humeral heads were delineated on all layers of the CT scans. 
Plan evaluation. The present study used a dose volume histogram (DVH) to evaluate the quality of the radiation plan. For the CTV and PTV, $D_{x}$ represents the minimum dose delivered to $x \%$ of the target volume and $V_{x}$ represents the volume receiving no less than $x \%$ of the prescription dose $(29,30)$. In addition, $D_{\min }, D_{\max }$ and $D_{\text {mean }}$ of the CTV and PTV represent the minimum, maximum and mean point dose of the target volume, respectively.

The conformity index (CI) and the homogeneity index (HI) of the PTV were automatically evaluated by the Monaco TPS to assess the PTV coverage rate. The CI indicates the ratio between the PTV and the irradiated volume at the prescription dose, and the HI implies the uniformity of the dose distribution in the target volume (31). The computational formulas of the $\mathrm{CI}$ and $\mathrm{HI}$ were as follows: $\mathrm{CI}=T V_{1}^{2} / T V \times V R_{1}$, where $T V_{1}$ represents the volume of the target that received the prescription dose, $T V$ represents the target volume and $V R 1$ represents the total volume of the prescription isodose. Notably, values of CI closer to 1.0 represent a better dose conformity of the PTV. HI $=D_{\max } / D_{\min }$, where $D_{\max }$ represents the maximum point dose and $D_{\min }$ represents the minimum point dose of the target volume. Notably, values of HI closer to 1.0 indicate a plan with less heterogeneity. Definitions of $D_{x}, D_{\min }, D_{\max }$ and $D_{\text {mean }}$ for all OAR and $V_{x}$ for the C-B are similar to definitions for the target volume. Other $V_{x}$ represents the volume receiving no less than $x$ Gray (Gy) $(29,30)$.

Dose prescription. Intensity-modulated radiotherapy (IMRT) plans were performed with 6-mV x-ray, and 5-9 coplanar and isocenter radiation treatment fields for five groups of each patient. Subsequently, a dose of $50 \mathrm{~Gy}$ in 25 fractions of $2 \mathrm{~Gy}$ was prescribed to the PTV. Notably, $95 \%$ of the target volume should be included by $95 \%$ of the prescribed dose (4,750 centigray, cGy) and not $>5 \%$ should be encompassed by $105 \%$ of the prescribed dose (5,250 cGy). In the present study, the dose limits of OAR were as follows: For the ipsilateral lungs (I-L), $V_{20}<25 \%$ and $D_{\text {mean }}<15 \mathrm{~Gy}$; for the bilateral lungs, $V_{20}<20 \%$; and for the heart, $V_{30}<10 \%$ and $V_{40}<5 \%$.

Statistical analysis. The disease stage of patients was evaluated according to the 7th American Association of Cancer (AJCC) staging system (32). $D_{x}, V_{x}, \mathrm{CI}$ and $\mathrm{HI}$ were extracted using the Monaco system. All data were analysed using SPSS software (version 21.0; IBM Corp., Armonk, NY, USA) with a randomised block design. The Shapiro-Wilk test and Levene test were used to evaluate the normality and homogeneity of data. Data that are normaly distributed are expressed as mean \pm standard deviation and presented as bar plots; all other data are expressed as median (interquartile range) and presented as box and whisker plots (Figs. 1-9). For each evaluation index, analysis of variance followed by a Least Significant Difference test were used when data satisfied normal distribution and homoscedasticity. Otherwise, a Friedman test and pairwise comparison were used. $\mathrm{P}<0.05$ was considered to indicate a statistically significant difference.

\section{Results}

Dosage comparison of the CTV and PTV between the 3D-CT plan and the 4D-CT plan. The present study compared the dosimetric characteristics between the 3D-CT radiotherapy plan and four different 4D-CT radiotherapy plans (AIP, MIP, T00 and T50) in the same order for the seven patients with residual breast tissue. For target dose parameters, no significant difference was observed in the $\mathrm{CI}$ and $\mathrm{HI}$ of the PTV between the 3D-CT and 4D-CT plans (Table II; Fig. 1). In addition, target volumes (including the total volume, $V_{100}, V_{95}, V_{90}$ and $V_{50}$ ) of the CTV and PTV of the 4D-CT plan were slightly lower compared with the 3D-CT plan (Table II; Figs. 2 and 3). Furthermore, $D_{\min }, D_{\max }$ and $D_{\text {mean }}$ of the CTV and PTV in the MIP and AIP plans were slightly higher compared with that of the 3D-CT plan (Table II; Figs. 2 and 3).

Dosage comparison of OAR between the 3D-CT and 4D-CT plans. In the present study, the following comparisons of dose parameters of OAR were made. For the C-B (Table III; Fig. 4), the total volume of the 4D-CT plan was markedly lower compared with the $3 \mathrm{D}-\mathrm{CT}$ plan. In addition, $D_{\min }, D_{\max }$ and $D_{\text {mean }}$ of the AIP plan were lower than those of $3 \mathrm{D}-\mathrm{CT}$ and MIP plans. No marked differences were observed in dose parameters between the MIP and AIP plans. For the I-L (Table III; Fig. 5), volumes (including the total volume, $V_{30}$, $V_{20}$, and $V_{10}$ ) of the T00 plan were the highest, followed by the 3D-CT and AIP plans, and the volumes of the T50 and MIP plans were the lowest. For the contralateral lungs (C-L) (Table III; Fig. 6), the total volumes of the MIP plan were markedly lower compared with that of the 3D-CT, AIP and T00 plans; however, the volume, of the T00 plan were higher compared with the T50 plan. For the I-L and C-L (Table III; Figs. 5 and 6), no statistical differences were observed in the dosage among the five plans. In addition, for the contralateral and ipsilateral humeral head (Table III; Fig. 7), no significant differences were observed in dose parameters between the 3D-CT and 4D-CT plans. For the heart (Table III; Figs. 8 and 9), regardless of whether breast lesions were in the right or left side, the volume of the MIP and AIP plans were slightly higher compared with that of the 3D-CT plan, with no significant difference in dose among the 3D-CT, MIP and AIP plans. When breast lesions were on the left side, for the heart, $V_{40}$ and $V_{30}$ of the MIP and AIP plans were slightly lower compared with those of the 3D-CT plan (Table III; Fig. 8).

\section{Discussion}

Previous studies have established that radiotherapy based on 4D-CT simulation enhances the accuracy of dosage and determines the locations of tumour(s) and OAR in the chest and abdomen (9-26,33). Certain previous studies have compared the composite methods of 4D-CT radiotherapy for lung cancer and liver cancer $(9,17,20,23-26,33)$; however, at present, to the best of our knowldege, there is no study that has been published in English, which has investigated the same for breast cancer. To the best of the our knowledge, the present study is the first to compare the target volume and dosimetric differences between the 3D-CT radiotherapy plan and four 4D-CT radiotherapy plans (T00, T50, MIP and AIP plans) for whole breast radiotherapy.

For dose parameters of targets, all target volumes (including the total volume, $V_{100}, V_{95}, V_{90}$ and $V_{50}$ ) of the CTV and PTV in the 3D-CT plan were slightly higher compared with four 4D-CT 

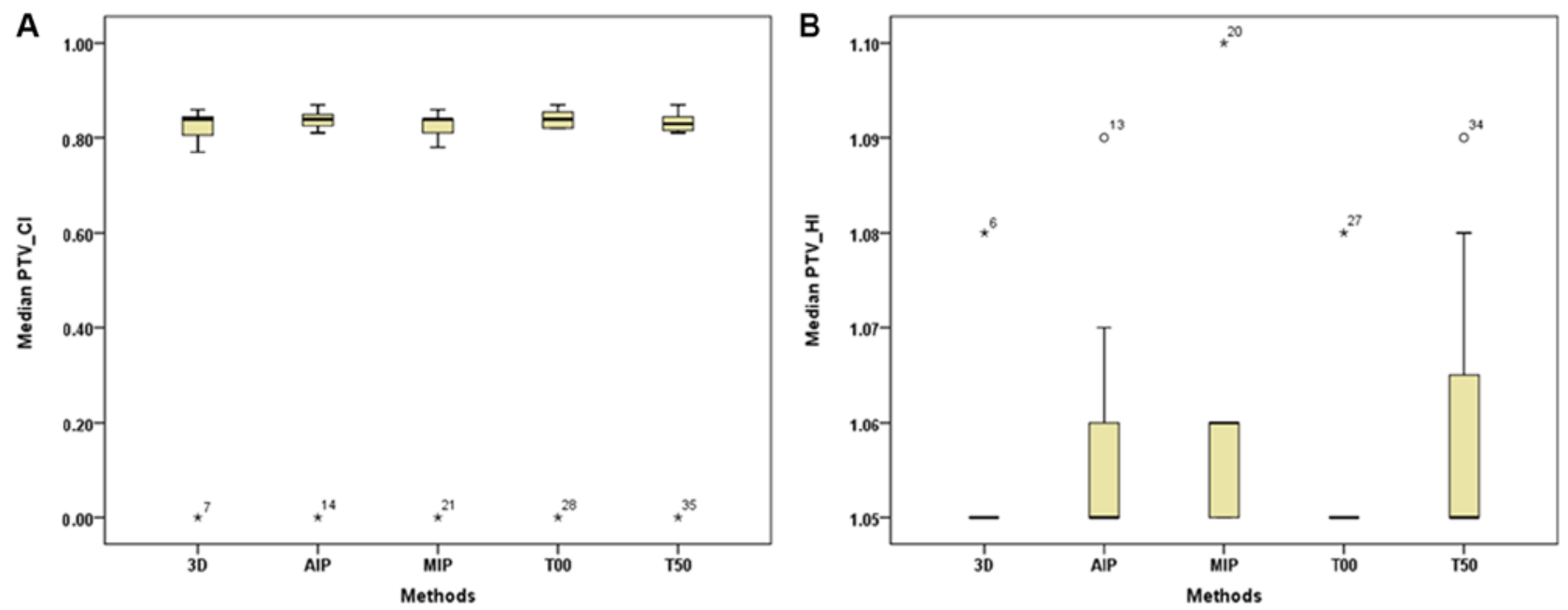

Figure 1. CI and $\mathrm{HI}$ values for the 3D computerised tomography and four-dimensional computerised tomography plans in 7 patients. (A) CI values. (B) HI values. The horizontal axis represents the five different plans and the vertical axis represents the median values of the CI and $\mathrm{HI}$. CI, conformity index; HI, homogeneity index; AIP, average intensity projection; MIP, maximal intensity projection; T00, end of inhalation; T50, end of exhalation; 3D, three-dimensional; Gy, gray.
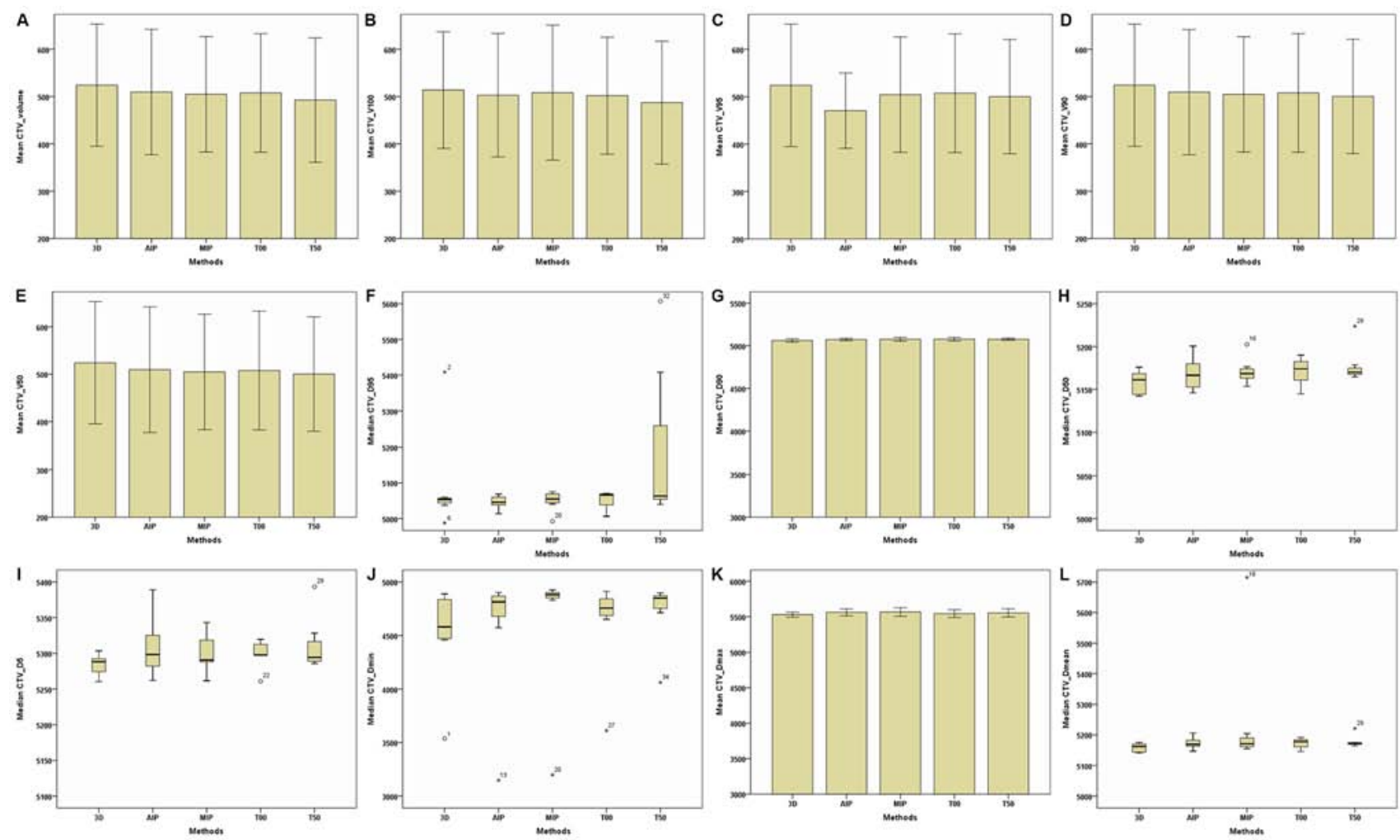

Figure 2. Parameters of the CTV between the 3D and four-dimensional computerised tomography plans in seven patients. (A) CTV-Volume. (B) CTV-V ${ }_{100}$.

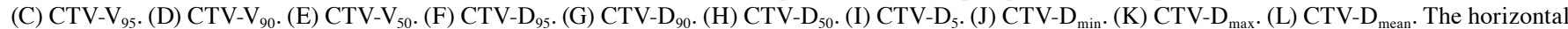
axis represents five different plans and the vertical axis represents the mean or median dose size. CTV, clinical target volume; 3D, three-dimensional; AIP, average intensity projection; MIP, maximal intensity projection; T00, end of inhalation; T50, end of exhalation; $D_{x}$, the minimum dose delivered to $x \%$ of the target volume; $V_{x}$, the volume receiving no less than $x \%$ of the prescription dose; $D_{\max }$, maximum point dose; $D_{\min }$, minimum point dose of the target volume; $D_{\text {mean }}$, mean point dose of the target volume; Gy, gray.

plans, with no difference between the T00 and T50 plans. For the $\mathrm{C}-\mathrm{B}$, the total volume in the $3 \mathrm{D}-\mathrm{CT}$ plan was markedly higher compared with the 4D-CT plan. Therefore, it could be concluded that breast tissues exert little impact on the respiration movement, which suggests that the volume changes of the breasts should be ignored (34). In addition, differences in target volumes between the 3D-CT and 4D-CT plans were not due to respiration movement-caused displacement. In free breathing, partial target areas of the 3D-CT plan were scanned recurrently or missed due to uncertainty in the beginning time of the scan and the whole scan time was longer compared with the 4D-CT scan; therefore, $4 \mathrm{D}-\mathrm{CT}$ images evaluated the location, size and 

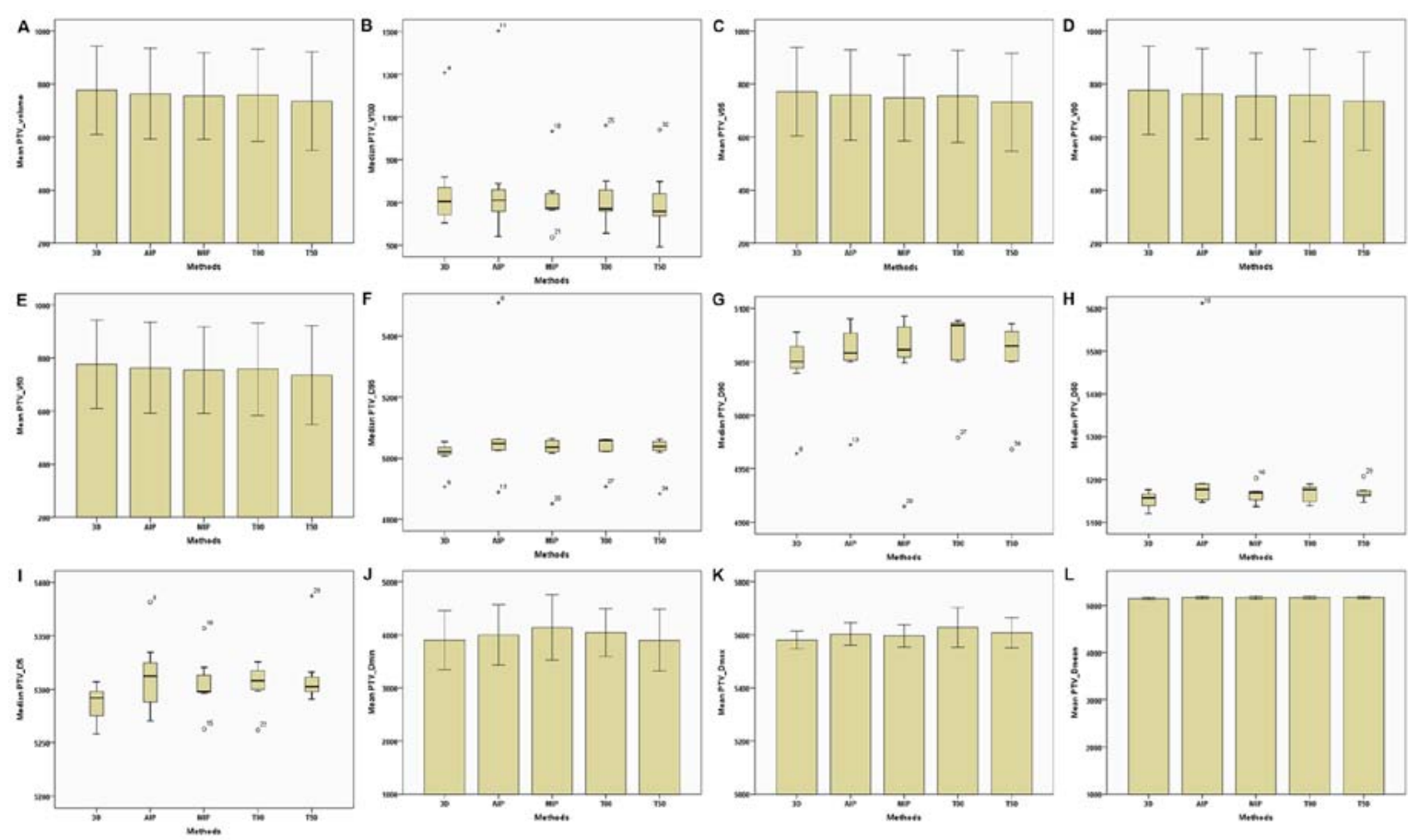

Figure 3. Parameters of the PTV between the 3D and four-dimensional computerised tomography plans in seven patients. (A) PTV-Volume. (B) PTV-V ${ }_{100}$. (C)

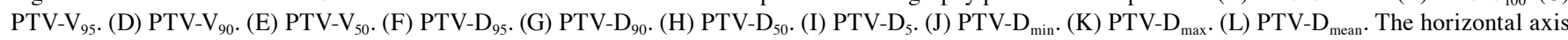
represents the five different plans and the vertical axis represents the mean or median dose size. PTV, plan target volume; 3D, three-dimensional; AIP, average intensity projection; MIP, maximal intensity projection; T00, end of inhalation; T50, end of exhalation; $D_{x}$, the minimum dose delivered to $x \%$ of the target volume; $V_{x}$, the volume receiving no less than $x \%$ of the prescription dose; $D_{\max }$, maximum point dose; $D_{\min }$, minimum point dose of the target volume; $D_{\text {mean }}$, mean point dose of the target volume; Gy, gray.
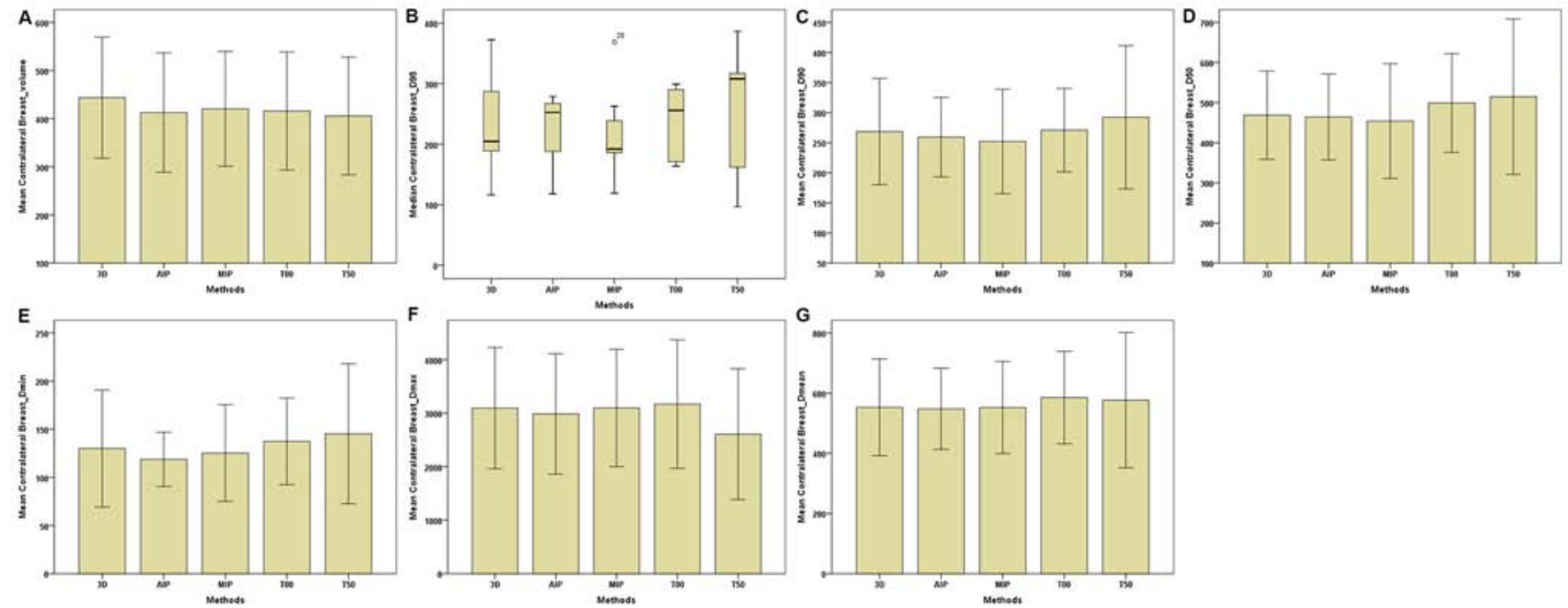

Figure 4. Parameters of the contralateral breast between the 3D and four-dimensional computerised tomography plans in seven patients. (A) Contralateral

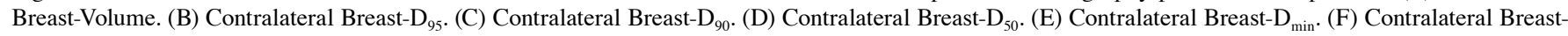
$\mathrm{D}_{\max }$ (G) Contralateral Breast- $\mathrm{D}_{\text {mean }}$. The horizontal axis represents the five different plans and the vertical axis represents the mean or median dose size. $3 \mathrm{D}$, three-dimensional; AIP, average intensity projection; MIP, maximal intensity projection; T00, end of inhalation; T50, end of exhalation; $D_{x}$, the minimum dose delivered to $x \%$ of the target volume; $V_{x}$, the volume receiving no less than $x \%$ of the prescription dose; $D_{\max }$, maximum point dose; $D_{\min }$, minimum point dose of the target volume; $D_{\text {mean }}$, mean point dose of the target volume; Gy, gray.

shape of targets more precisely (35). However, Bedi et al (36) reported no significant differences in the left breast volume contoured on a 3D-CT scan $\left(1,005 \pm 559 \mathrm{~cm}^{3}\right), 4 \mathrm{D}$-CT scan with full inspiration phase $\left(1,019 \pm 563 \mathrm{~cm}^{3}\right), 4 \mathrm{D}-\mathrm{CT}$ scan with full expiration phase $\left(1,023 \pm 573 \mathrm{~cm}^{3}\right)$ and $4 \mathrm{D}-\mathrm{CT}$ scan derived by AIP $\left(1023 \pm 573 \mathrm{~cm}^{3}\right)$. The difference between the two studies could be attributed to the following: i) Breast locations in western females are more easily affected by the respiration movement, as the breast size of western females is typically larger compared with Chinese females; and ii) all breast lesions in the previous study by Bedi et al (36) were in the left chest where the impact of the cardiac motion could not be ignored; however, in the present study, the number of lesions on the right side was greater than that on the left side (right-to-left ratio, 4:3) 

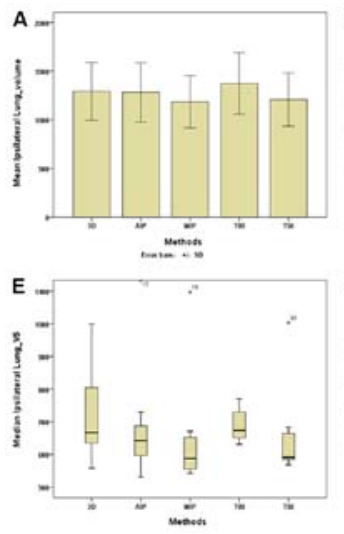

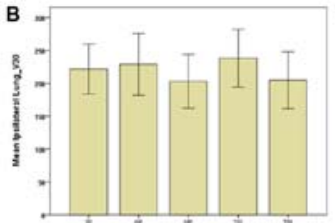

$\ldots$

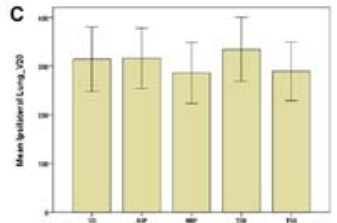

-
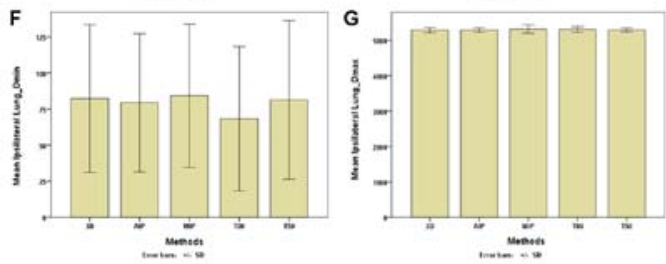
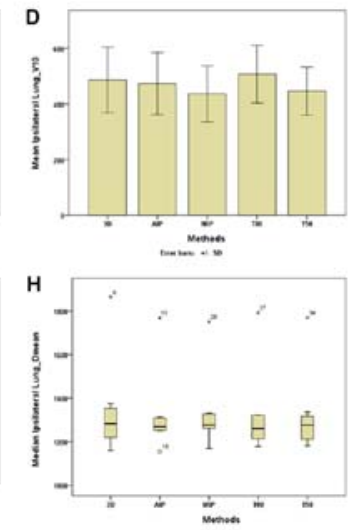

Figure 5. Parameters of the ipsilateral lungs between the 3D and four-dimensional computerised tomography plans in seven patients. (A) Ipsilateral Lungs-Volume. (B) Ipsilateral Lungs- $\mathrm{V}_{30}$. (C) Ipsilateral Lungs- $\mathrm{V}_{20}$. (D) Ipsilateral Lungs- $\mathrm{V}_{10}$. (E) Ipsilateral Lungs- $\mathrm{V}_{5}$. (F) Ipsilateral Lungs- $\mathrm{D}_{\min }$. (G) Ipsilateral Lungs- $\mathrm{D}_{\text {max }}$. (H) Ipsilateral Lungs- $\mathrm{D}_{\text {mean }}$. The horizontal axis represents the five different plans and the vertical axis represents the mean or median dose size. 3D, three-dimensional; AIP, average intensity projection; MIP, maximal intensity projection; T00, end of inhalation; T50, end of exhalation; $D_{x}$, the minimum dose delivered to $x \%$ of the target volume; $V_{x}$ represents the volume receiving no less than $x$ Gy; $D_{\max }$, maximum point dose; $D_{\min }$, minimum point dose of the target volume; $D_{\text {mean }}$, mean point dose of the target volume; Gy, gray.
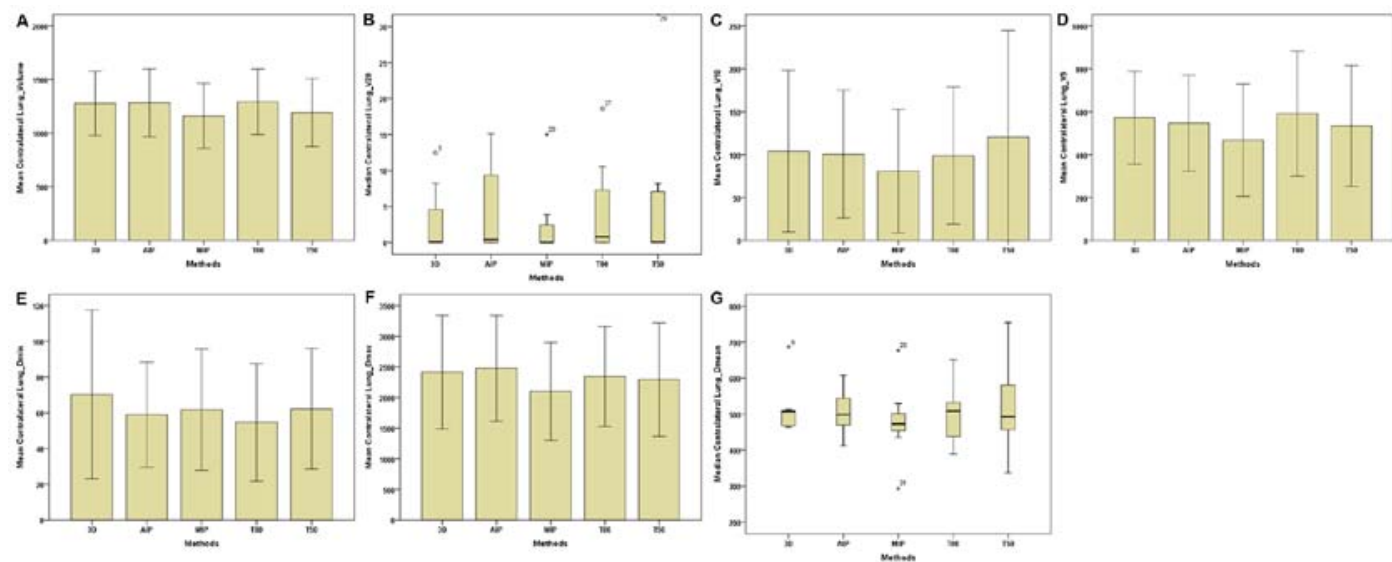

Figure 6. Parameters of the contralateral lungs between the 3D and four-dimensional computerised tomography plans in seven patients. (A) Contralateral Lungs-Volume. (B) Contralateral Lungs- $\mathrm{V}_{20}$. (C) Contralateral Lungs- $\mathrm{V}_{10}$. (D) Contralateral Lungs- $\mathrm{V}_{5}$. (E) Contralateral Lungs- $\mathrm{D}_{\min }$. (F) Contralateral Lungs$\mathrm{D}_{\max }$. $(\mathrm{G})$ Contralateral Lungs- $\mathrm{D}_{\text {mean }}$. The horizontal axis represents the five different plans and the vertical axis represents the mean or median dose size. 3D, three-dimensional; AIP, average intensity projection; MIP, maximal intensity projection; T00, end of inhalation; T50, end of exhalation; $D_{x}$, the minimum dose delivered to $x \%$ of the target volume; $V_{x}$, represents the volume receiving no less than $x$ Gy; $D_{\max }$, maximum point dose; $D_{\min }$, minimum point dose of the target volume; $D_{\text {mean }}$, mean point dose of the target volume; Gy, gray.
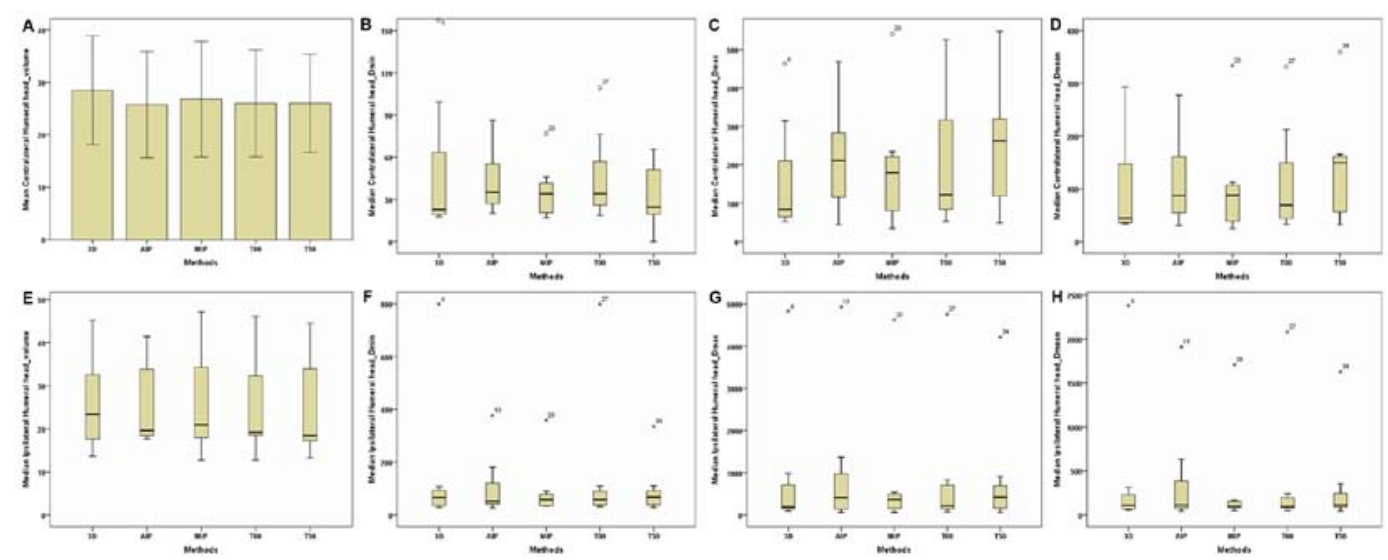

Figure 7. Parameters of the contralateral and ipsilateral humeral head between the 3D and four-dimensional computerised tomography plans in seven patients. (A) Contralateral Humeral Head-Volume. (B) Contralateral Humeral Head- $\mathrm{D}_{\min }$. (C) Contralateral Humeral Head-D $\mathrm{m}_{\max }$. (D) Contralateral Humeral Head-D mean $_{\text {. }}$ (E) Ipsilateral Humeral Head-Volume. (F) Ipsilateral Humeral Head-D min. $_{\text {. }}$ (G) Ipsilateral Humeral Head-D $D_{\max }$. (H) Ipsilateral Humeral Head-Dmean. The horizontal axis represents the five different plans and the vertical axis represents the mean or median dose size. 3D, three-dimensional; AIP, average intensity projection; MIP, maximal intensity projection; T00, end of inhalation; T50, end of exhalation; $D_{x}$, the minimum dose delivered to $x \%$ of the target volume; $V_{x}$ represents the volume receiving no less than $x \mathrm{~Gy} ; D_{\max }$, maximum point dose; $D_{\min }$, minimum point dose of the target volume; $D_{\text {mean }}$, mean point dose of the target volume; Gy, gray. 

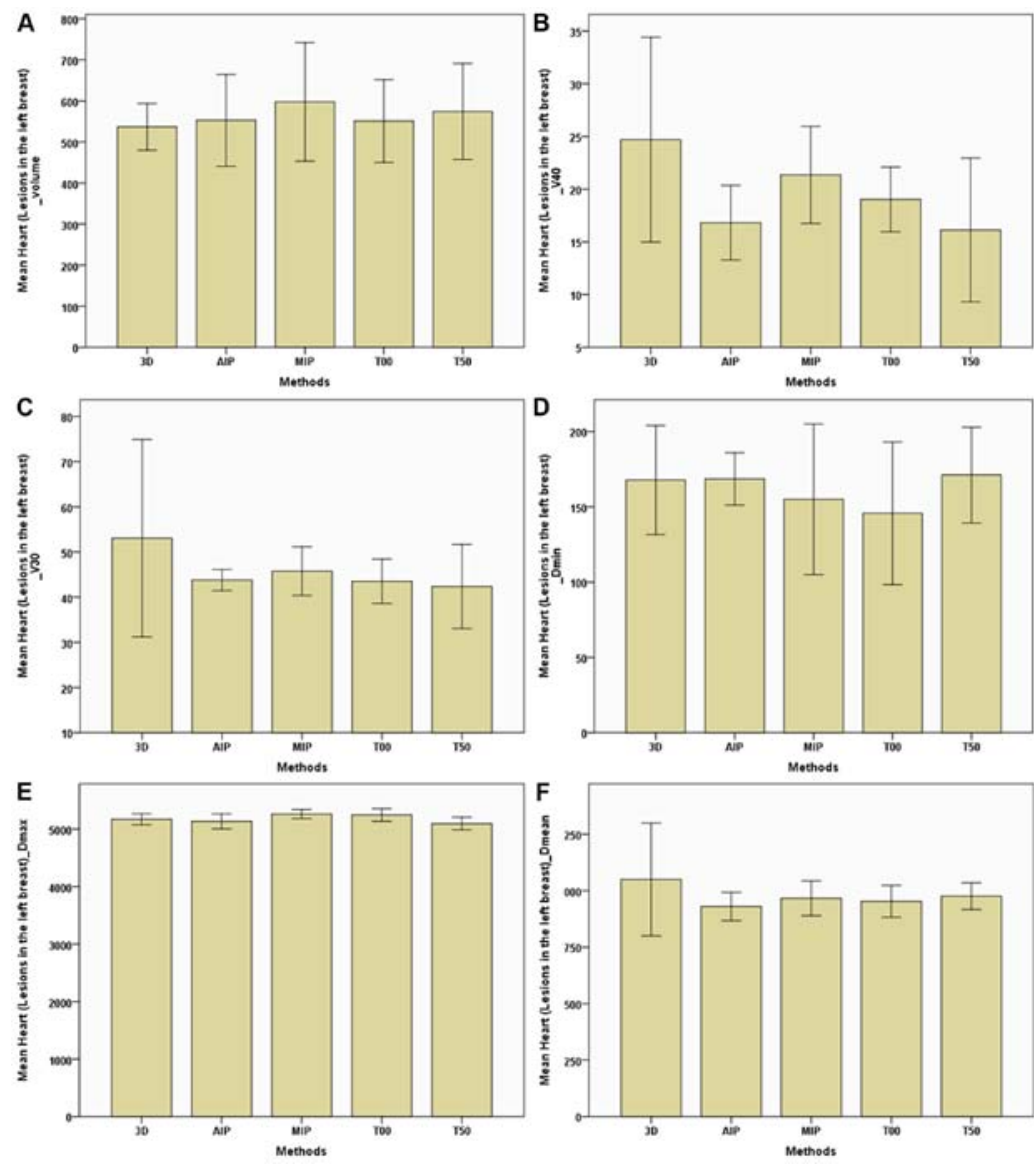

Figure 8. Parameters of the heart (breast lesions in the left) between the 3D and four-dimensional computerised tomography plans in seven patients. (A) Heart (lesions in the left breast)-Volume. (B) Heart (lesions in the left breast)- $\mathrm{V}_{40}$. (C) Heart (lesions in the left breast)- $\mathrm{V}_{30}$. (D) Heart (lesions in the left breast)- $\mathrm{D}_{\text {min. }}$ (E) Heart (lesions in the left breast)- $\mathrm{D}_{\max }$. (F) Heart (lesions in the left breast)- $\mathrm{D}_{\text {mean }}$. The horizontal axis represents the five different plans and the vertical axis represents the mean or median dose size. 3D, three-dimensional; AIP, average intensity projection; MIP, maximal intensity projection; T00, end of inhalation; T50, end of exhalation; $D_{x}$, the minimum dose delivered to $x \%$ of the target volume; $V_{x}$, represents the volume receiving no less than $x$ Gy; $D_{\max }$, maximum point dose; $D_{\text {min }}$, minimum point dose of the target volume; $D_{\text {mean }}$, mean point dose of the target volume; Gy, gray.
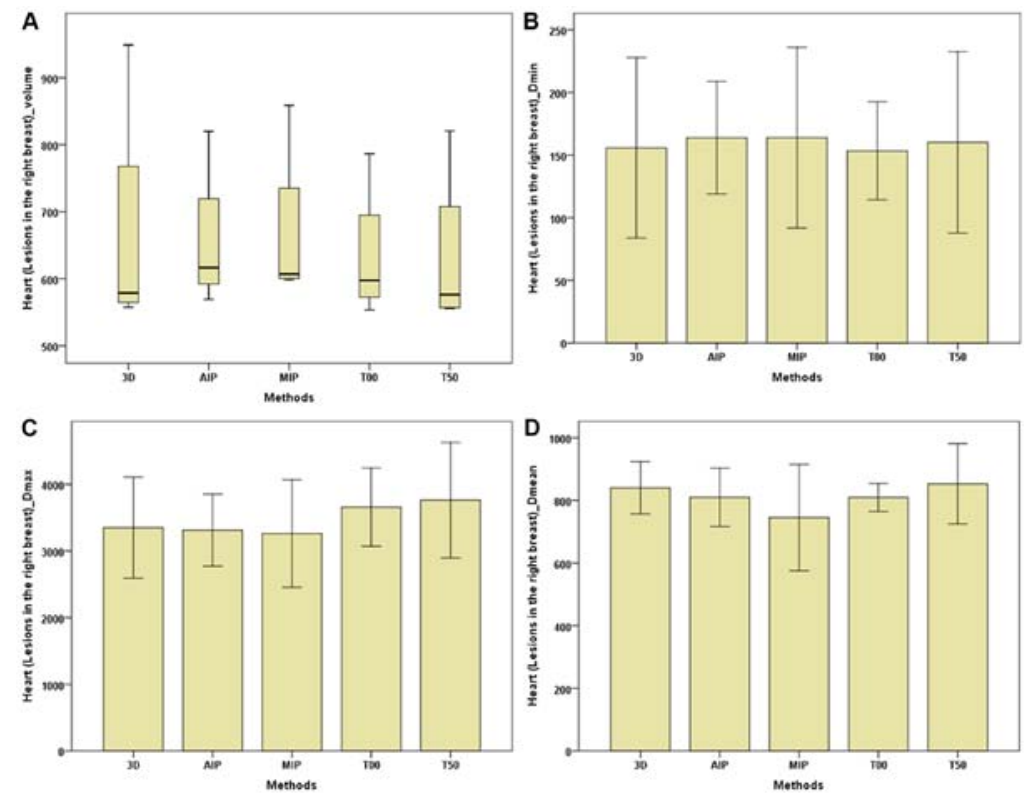

Figure 9. Parameters of the heart (breast lesions in the right) between the 3D and four-dimensional computerised tomography plans in seven patients. (A) Heart (lesions in the right breast)-Volume. (B) Heart (lesions in the right breast)- $\mathrm{D}_{\min }$. (C) Heart (lesions in the right breast)- $\mathrm{D}_{\text {max }}$. (D) Heart (lesions in the right breast)$\mathrm{D}_{\text {mean }}$. The horizontal axis represents the five different plans and the vertical axis represents the mean or median dose size. 3D, three-dimensional; AIP, average intensity projection; MIP, maximal intensity projection; T00, end of inhalation; T50, end of exhalation; $D_{x}$, the minimum dose delivered to $x \%$ of the target volume; $V_{x}$, represents the volume receiving no less than $x \mathrm{~Gy} ; D_{\max }$, maximum point dose; $D_{\min }$, minimum point dose of the target volume; $D_{\operatorname{mean}}$, mean point dose of the target volume; Gy, gray. 

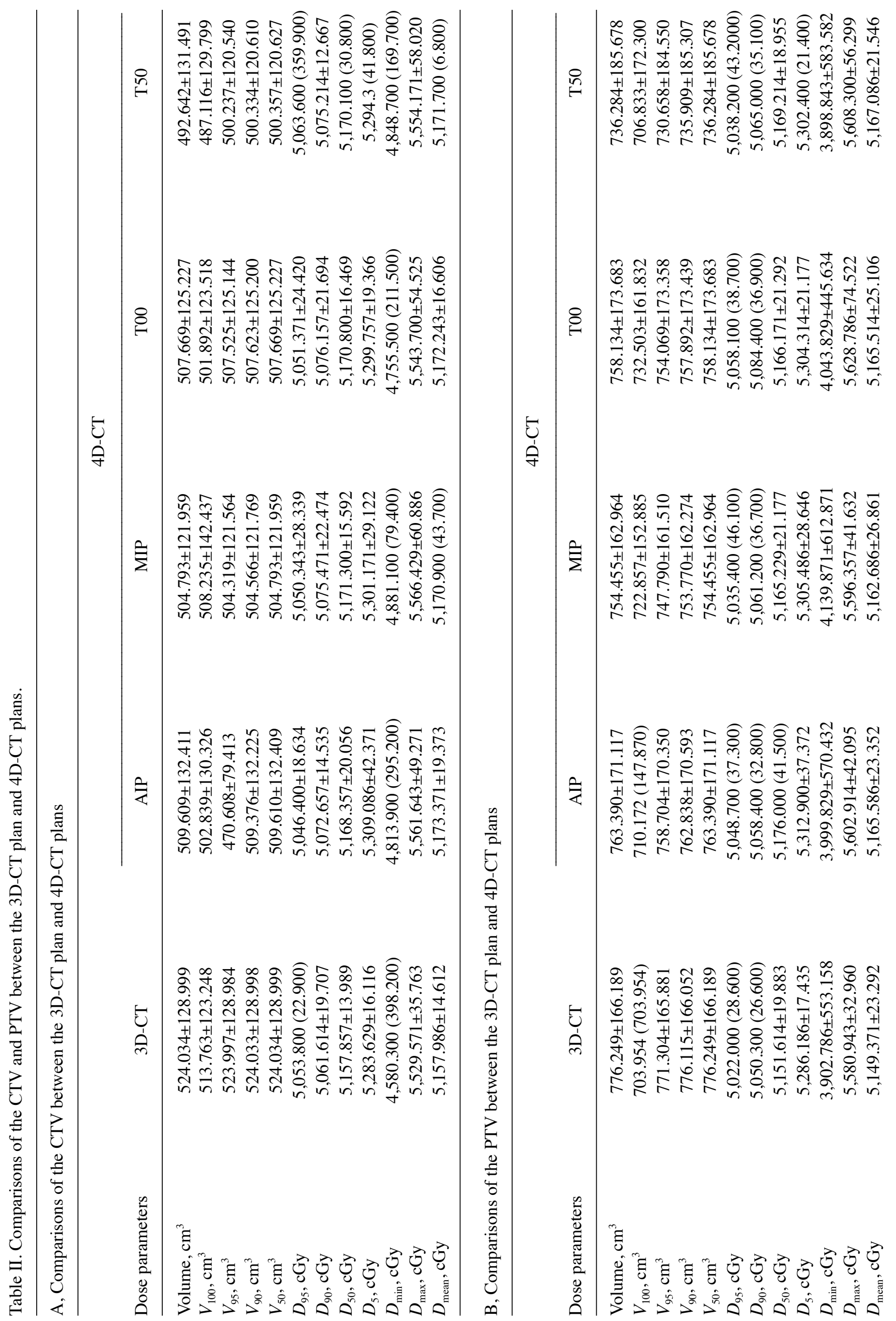
Respiration movements not only affect displacements of target volumes but also exert a considerable impact on the radiation dose distribution (37-39). In the clinical setting, during the process of treatment, changes in the dose distribution caused by target bias in the 3D-CT plan cannot satisfy the treatment requirements, which may increase the risk of local recurrence. In the present study, $D_{\min }, D_{\text {max }}$ and $D_{\text {mean }}$ of the CTV and PTV in the MIP and AIP plans were slightly higher compared with that of the 3D-CT plan. However, $D_{\min }, D_{\max }$ and $D_{\text {mean }}$ of the $\mathrm{C}-\mathrm{B}$ in the 3D-CT and T00 plans were the highest and those of the AIP and MIP plans were the lowest. In addition, no marked difference was observed in dose parameters between the MIP and AIP plans, indicating that the dose distribution of the MIP and AIP plans is better compared with that of the 3D-CT plan. In addition, this suggests that the MIP and AIP plans can prevent and decrease radiation exposure to normal breast. In the present study, no marked differences were observed in the $\mathrm{CI}$ and $\mathrm{HI}$ between the 3D-CT and four 4D-CT plans. As the target volumes in the five groups were similar, all plans were achieved by IMRT with the same pattern. Reportedly, the IMRT technique achieved improved CI and HI compared with 3D conformal radiotherapy, and increased OAR sparing and decreased the late effects, which enhanced the quality of life of the patients $(31,40,41)$.

Previous studies have compared different composite methods for lung cancer and liver cancer (21,23-28). Zhao et al (27) reported that lung volumes of the AIP plan were close to the 3D-CT volume in lung cancer and that of the MIP plan was smaller compared with the AIP plan by $11.4 \pm 2.3 \%$. In addition, the DVH of the MIP plan revealed that the MIP plan was less sensitive to breathing movements (27). However, Simon et al (28) reported that the internal target volume of the MIP plan was closer to the actual volume compared with that of the AIP plan. Similarly, a previous study determined that the GTV of the MIP plan was markedly higher compared with that of the AIP plan for stereotactic body radiotherapy planning in lung cancer (20). The differences in the aforementioned studies may have resulted from different evaluated standard and research methods. MIP images are acquired by finding the maximum $\mathrm{CT}$ value of images from all respiratory phases. A previous study reported that MIP images include the motion and position extent of a lung tumour as the density of a tumour was higher compared with that of the surrounding normal lung tissue (42). However, Mohatt et al (25) reported that in clinical lung tumour cases with displacements ranging between 0.1 and $2.2 \mathrm{~cm}$, the MIP plan typically underestimated target volumes and resulted in a PTV ratio of $0.95 \pm 0.15$. When tumours were located close to the chest wall, MIP images were more easily affected by the surrounding structures with high or equal density, including the cartilage tissues and muscles, compared with AIP images $(22,27)$. Park et al (43) reported that the MIP plan was markedly different from the ten-phase fusion plan when breathing was irregular or a tumour was close to similar-density tissues. However, in the present study, no marked dosimetric difference was observed between the MIP and AIP plans, which could be attributed to the fact that breast tissue movements are less sensitive to breathing; therefore, the impact of MIP images caused by surrounding structures was low.

In addition to improving the sparing of the $\mathrm{C}-\mathrm{B}$, another objective of breast radiotherapy is to 

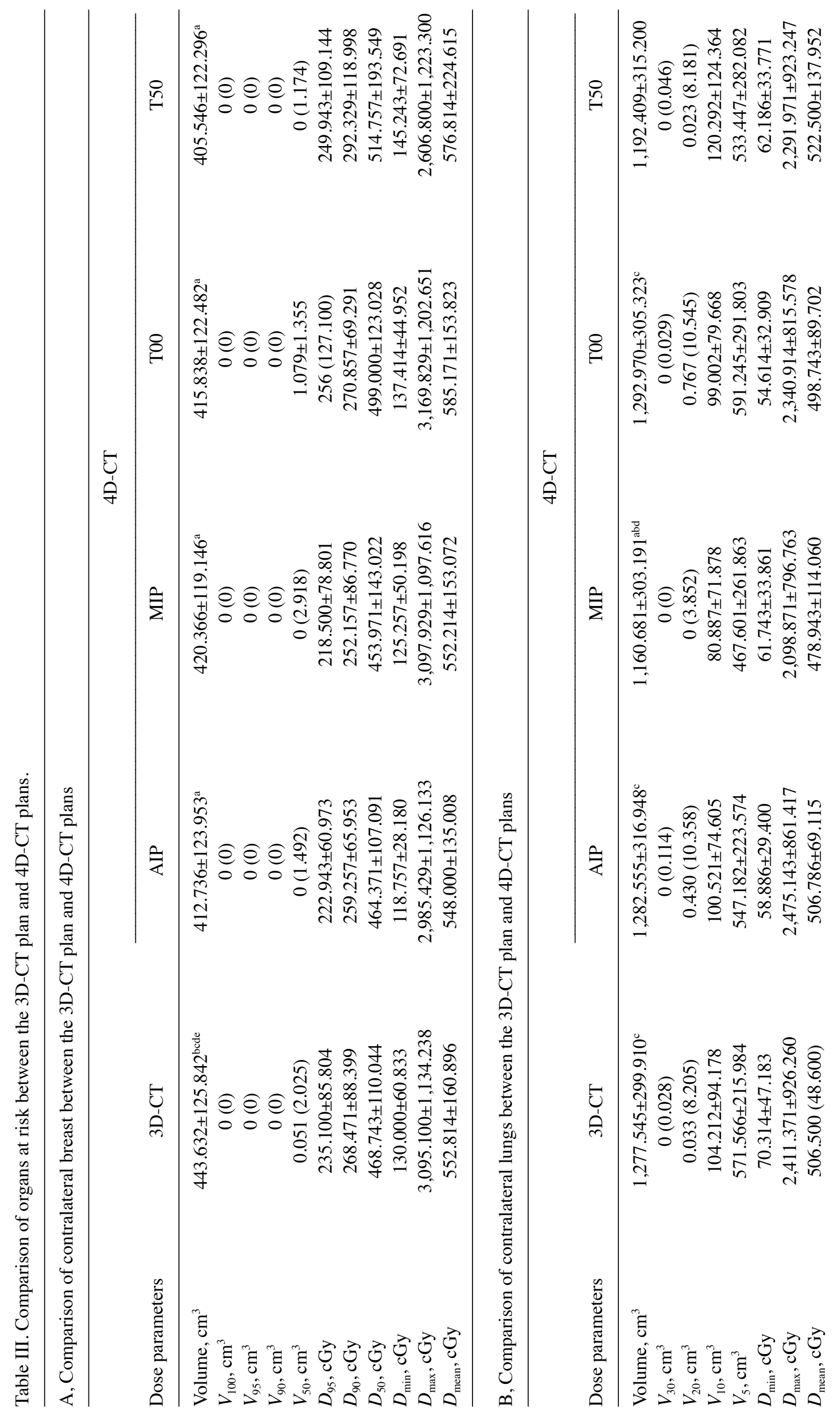

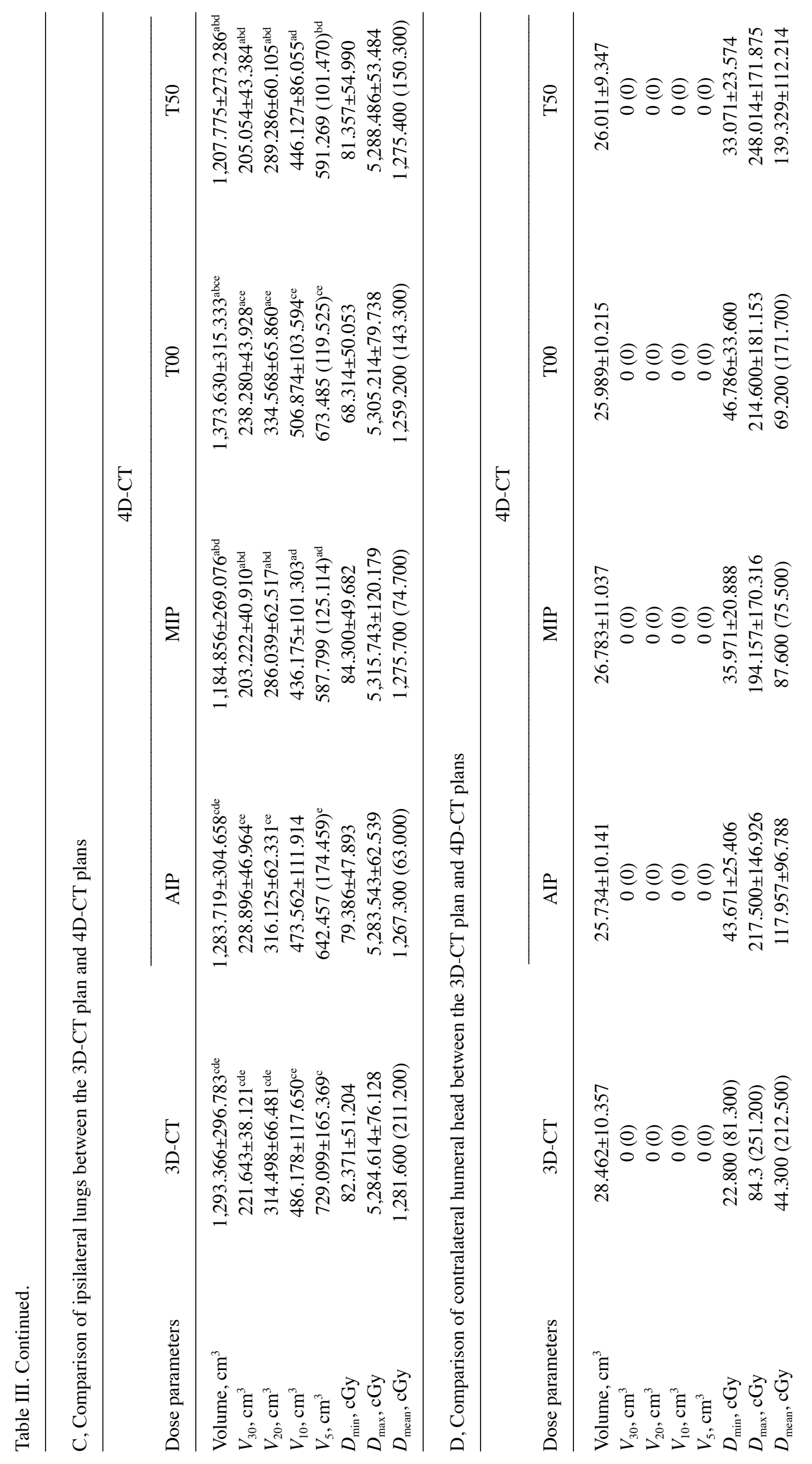

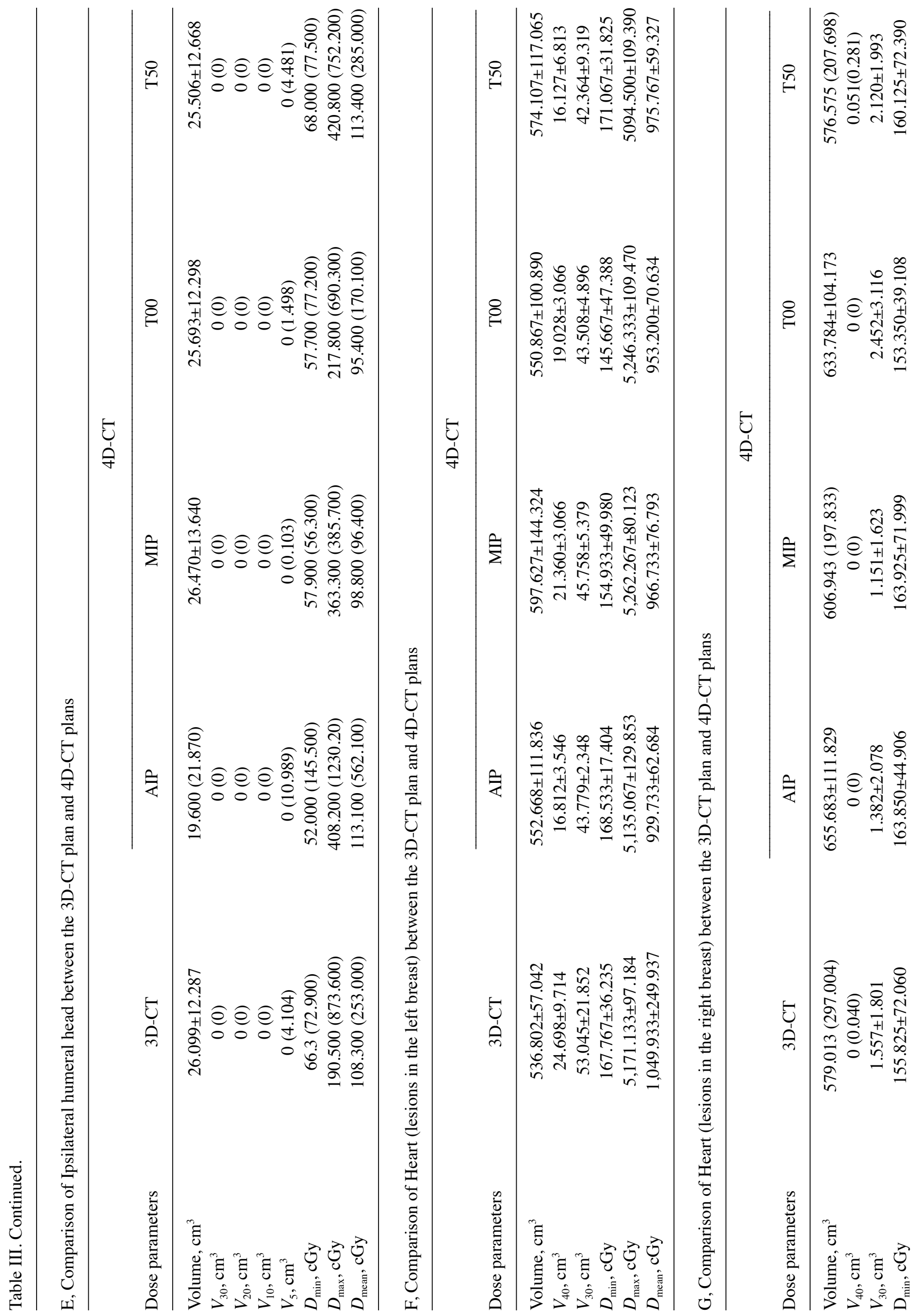
decrease the irradiation dose and volume of the heart and bilateral lungs. In the present study, for the I-L, marked differences were identified in volumes (including the total volume, $V_{30}, V_{20}, V_{10}$ and $V_{5}$ ) among the five plans (3D-CT, T00, T50, MIP and AIP). Additionally, volumes of the T00 plan were the highest, followed by the 3D-CT and AIP plans, and those of the T50 and MIP plans were the lowest. For the C-L, total volumes of the MIP plan were markedly lower compared with those of the 3D-CT, AIP and T00 plans, and total volumes of the T00 plan were higher compared with those of the T50 plan. Whether in the I-L or C-L, no statistical difference of dosage was observed among the five plans, indicating that in free breathing, although no apparent breast displacements occurred between the two extreme respiratory phases, the sufficiently apparent difference of bilateral lungs volumes resulting from thoracic movements were easily observed. Total volumes of the lungs in the MIP plan were easily affected by the surrounding structures with high or equal density $(22,27)$, which induced smaller volumes in the MIP plan compared with others and then induced the smallest $V_{30}, V_{20}, V_{10}$ and $V_{5}$. Therefore, the present study could not completely establish that the MIP plan is superior to the others. For the heart, dosimetric parameters of plans are affected not only by respiratory movements but also by their own rhythm. In the present study, whether breast lesions were in the right or left side, heart volumes of the MIP and AIP plans were slightly higher compared with that of the 3D-CT plan, with no marked differences in dose among the 3D-CT, MIP and AIP plans. However, for hearts of patients with lesions in the left breast, $V_{40}$ and $V_{30}$ of the MIP and AIP plans were slightly lower compared with that of the $3 \mathrm{D}-\mathrm{CT}$ plan. These results indicated that the MIP and AIP plans may improve sparing of the heart, particualy lesions in the left side, and the I-L. However, Bedi et al (36) reported that dosimetric results for the heart and the I-L exhibited no statistically significant differences between the 3D-CT and 4D-CT plans for patients with left-sided breast cancer, and that improved sparing of the heart and the lungs could only be attained by decreasing the posterior margins of the breast target volumes. For contralateral and ipsilateral humeral heads, no marked differences were observed in dose parameters between the 3D-CT and 4D-CT plans as humeral heads were far away from the targets and could not be affected by therapy plans.

In conclusion, for whole breast radiotherapy of breast cancer with residual tissues (including postoperative radiotherapy, neoadjuvant radiotherapy and palliative radiotherapy), 4D-CT radiotherapy techniques based on the MIP and AIP plans provide a slightly smaller radiation area and slightly higher radiotherapy dosage of the CTV and PTV compared with 3D-CT radiotherapy. For the C-B, the dose distribution in the MIP and AIP plans is better compared with the 3D-CT plan; therefore, MIP and AIP plans prevent and reduce radiation exposure to normal breast. The MIP and AIP plans also improve sparing of the heart (particularly breast lesions in the left side) and the I-L. Furthermore, the dosimetric differences between the MIP and AIP plans are not significant. Therefore, these plans are worth considering for whole breast radiotherapy.

\section{Acknowledgements}

Not applicable. 


\section{Funding}

The present study was supported by the National Natural Science Foundations of China (grant nos. 81772793/ H1621, 31201060/C0709, 30973175/C1701 and 81172490/ H1621), Programme for New Century Excellent Talents in University (grant no. NCET-12-0440), Scientific and Technological Research Foundation of Shaanxi Province (grant no. 2012K13-01-06), Research Foundation of Health Department of Shaanxi Province (grant no. 2010D41), Qing Nian Jiao Shi Gen Zong Ji Hua of Xi'an Jiaotong University ('The Fundamental Research Funds for the Central Universities' awarded to to JR) and the Clinical Research Award of the First Affiliated Hospital of Xi'an Jiaotong University (grant no. XJTU1AHCR2014-041).

\section{Availability of data and materials}

All data generated or analysed during the present study are included in this published article.

\section{Authors' contributions}

YY delineated the target area, performed the data analysis and drafted the manuscript. ZLu, ZLi and WL participated in the experiments, and performed the radiation plan and reviewing. SS, LT, XM, JL and ED participated in data collection and reviewed the manuscript. JR participated in experimental design, experimental operation, helped to draft the manuscript and approved the final version of the manuscript to be published.

\section{Ethics approval and consent to participate}

The present study was approved by the Ethics Committee of First Affiliated Hospital of Xi'an Jiaotong University (Xi'an, China; approval no. 2015-101). Written informed consent was obtained from all participants.

\section{Patient consent for publication}

Not applicable.

\section{Competing interests}

The authors declare that they have no competing interests.

\section{References}

1. Kunkler IH, Williams LJ, Jack WJ, Cameron DA and Dixon JM; PRIME II investigators: Breast-conserving surgery with or without irradiation in women aged 65 years or older with early breast cancer (PRIME II): A randomised controlled trial. Lancet Oncol 16: 266-273, 2015.

2. Chan TY, Tan PW and Tang JI: Intensity-modulated radiation therapy for early-stage breast cancer: Is it ready for prime time? Breast Cancer (Dove Med Press) 9: 177-183, 2017.

3. Keller LM, Sopka DM, Li T, Klayton T, Li J, Anderson PR, Bleicher RJ, Sigurdson ER and Freedman GM: Five-year results of whole breast intensity modulated radiation therapy for the treatment of early stage breast cancer: The fox chase cancer center experience. Int J Radiat Oncol 84: 881-887, 2012.

4. Castaneda SA and Strasser J: Updates in the treatment of breast cancer with radiotherapy. Surg Oncol Clin N Am 26: 371-382, 2017.
5. Poleszczuk J, Luddy K, Chen L, Lee JK, Harrison LB, Czerniecki BJ, Soliman H and Enderling H: Neoadjuvant radiotherapy of early-stage breast cancer and long-term disease-free survival. Breast Cancer Res 19: 75, 2017.

6. Coelho RC, Da Silva FDML, Do Carmo IML, Bonaccorsi BV and Faroni LD: Neoadjuvant radiotherapy in locally advanced breast cancer refractory to chemotherapy-a single institution experience in Brazil. Ann Oncol 26: 30-31, 2015.

7. Maher M, Campana F, Mosseri V, Dreyfus H, Vilcoq JR, Gautier C, Asselain B and Fourquet A: Breast-cancer in elderly women: A retrospective analysis of combined treatment with tamoxifen and once-weekly irradiation. Int J Radiat Oncol Biol Phys 31: 783-789, 1995.

8. Fakie N: Advanced breast cancer: A retrospective review comparing two palliative radiotherapy protocols used at Groote Schuur Hospital between 2010 and 2013. University of Cape Town, 2016.

9. Wu G, Lian J and Shen D: Improving image-guided radiation therapy of lung cancer by reconstructing $4 \mathrm{D}-\mathrm{CT}$ from a single free-breathing 3D-CT on the treatment day. Med Phys 39: 7694-7709, 2012.

10. Tan Z, Liu C, Zhou Y and Shen W: Preliminary comparison of the registration effect of 4D-CBCT and 3D-CBCT in image-guided radiotherapy of stage IA non-small-cell lung cancer. J Radiat Res 58: 854-861, 2017.

11. Low D: 4D imaging and 4D radiation therapy: A new era of therapy design and delivery. Front Radiat Ther Oncol 43: 99-117, 2011.

12. Wang JZ, Li JB, Qi HP, Li YK, Wang Y, Zhang YJ and Wang W: Effect of contrast enhancement in delineating GTV and constructing IGTV of thoracic oesophageal cancer based on 4D-CT scans. Radiother Oncol 119: 172-178, 2016.

13. Li F, Li J, Xing J, Zhang Y, Fan T, Xu M, Shang D, Liu T and Song J: Analysis of the advantage of individual PTVs defined on axial 3D CT and 4D CT images for liver cancer. J Appl Clin Med Phys 13: 4017, 2012.

14. Huang TC, Hsiao CY, Chien CR, Liang JA, Shih TC and Zhang GG: IMRT treatment plans and functional planning with functional lung imaging from 4D-CT for thoracic cancer patients. Radiat Oncol 8: 3, 2013.

15. Hoang JK, Reiman RE, Nguyen GB, Januzis N, Chin BB, Lowry $\mathrm{C}$ and Yoshizumi TT: Lifetime attributable risk of cancer from radiation exposure during parathyroid imaging: Comparison of 4D CT and parathyroid scintigraphy. AJR Am J Roentgenol 204: W579-W585, 2015.

16. Ding Y, Li J, Wang W, Wang S, Fan T, Xu M, Shao Q and Ma Z: Displacement of the lumpectomy cavity defined by surgical clips and seroma based on 4D-CT scan for external-beam partial breast irradiation after breast-conserving surgery: A comparative study. Br J Radiol 86: 20130416, 2013.

17. Zhang S, Lin S, Yu H, Zhang H, Zhang G and Han P: Application of AIP and MIP CT on individual GTV delineation for tumor moving with respiration. In: International conference on biomedical engineering and biotechnology, pp 736-739, 2012.

18. Park CK, Pritz J, Zhang GG, Forster KM and Harris EE: Validating fiducial markers for image-guided radiation therapy for accelerated partial breast irradiation in early-stage breast cancer. Int J Radiat Oncol Biol Phys 82: e425-e431, 2012.

19. Wang S, Li J, Wang W, Zhang Y, Li F, Fan T and Shang D: A study on the displacements of the clips in surgical cavity for external-beam partial breast irradiation after breast-conserving surgery based on 4DCT. J Radiat Res 53: 433-438, 2012.

20. Bradley JD, Nofal AN, El Naqa IM, Lu W, Liu J, Hubenschmidt J, Low DA, Drzymala RE and Khullar D: Comparison of helical, maximum intensity projection (MIP), and averaged intensity (AI) 4D CT imaging for stereotactic body radiation therapy (SBRT) planning in lung cancer. Radiother Oncol 81: 264-268, 2006.

21. Ezhil M, Vedam S, Balter P, Choi B, Mirkovic D, Starkschall G and Chang JY: Determination of patient-specific internal gross tumor volumes for lung cancer using four-dimensional computed tomography. Radiat Oncol 4: 4, 2009.

22. Pan T, Sun $X$ and Luo D: Improvement of the cine-CT based 4D-CT imaging. Med Phys 34: 4499-4503, 2007.

23. Lee SY, Lim S, Ma SY and Yu J: Gross tumor volume dependency on phase sorting methods of four-dimensional computed tomography images for lung cancer. Radiat Oncol J 35: 274-280, 2017.

24. Han CH, Sampath S, Schultheisss TE and Wong JYC: Variations of target volume definition and daily target volume localization in stereotactic body radiotherapy for early-stage non-small cell lung cancer patients under abdominal compression. Med Dosim 42: 116-121, 2017. 
25. Mohatt DJ, Keim JM, Greene MC, Patel-Yadav A, Gomez JA and Malhotra HK: An investigation into the range dependence of target delineation strategies for stereotactic lung radiotherapy. Radiat Oncol 12: 166, 2017.

26. Khamfongkhruea C, Thongsawad S, Tannanonta $\mathrm{C}$ and Chamchod S: Comparison of CT images with average intensity projection, free breathing, and mid-ventilation for dose calculation in lung cancer. J Appl Clin Med Phys 18: 26-36, 2017.

27. Zhao L, Sandison GA, Farr JB, Hsi WC and Li XA: Dosimetric impact of intrafraction motion for compensator-based proton therapy of lung cancer. Phys Med Biol 53: 3343-3364, 2008.

28. Simon L, Giraud P, Servois V and Rosenwald JC: Initial evaluation of a four-dimensional computed tomography system, using a programmable motor. J Appl Clin Med Phys 7: 50-65, 2006.

29. Deseyne P, Speleers B, De Neve W, Boute B, Paelinck L, Van Hoof T, Van de Velde J, Van Greveling A, Monten C, Post G, et al: Whole breast and regional nodal irradiation in prone versus supine position in left sided breast cancer. Radiat Oncol 12: 89, 2017.

30. Yin L, Wu H, Gong J, Geng JH, Jiang F, Shi AH, Yu R, Li YH, Han SK, Xu B and Zhu GY: Volumetric-modulated arc therapy vs. c-IMRT in esophageal cancer: A treatment planning comparison. World J Gastroenterol 18: 5266-5275, 2012.

31. Salimi M, Abi KST, Nedaie HA, Hassani H, Gharaati H, Samei M, Shahi R and Zarei H: Assessment and comparison of homogeneity and conformity indexes in step-and-shoot and compensator-based intensity modulated radiation therapy (IMRT) and Three-dimensional conformal radiation therapy (3D CRT) in prostate cancer. J Med Signals Sens 7: 102-107, 2017.

32. Lee JH, Eubank WB and Mankoff DA: Breast Cancer. In: Nuclear Oncology. Strauss H, Mariani G, Volterrani D and Larson S (eds). Springer, New York, NY, pp363-382, 2013.

33. Xing J, Li JB, Zhang YJ, Li FX, Fan TY, Xu M, Shang DP and Han JJ: Comparison of three methods to delineate internal gross target volume of the primary hepatocarcinoma based on four-dimensional CT simulation images. Zhonghua Zhong Liu Za Zhi 34: 122-128, 2012 (In Chinese).

34. Richter A, Sweeney R, Baier K, Flentje M and Guckenberger M: Effect of breathing motion in radiotherapy of breast cancer: 4D dose calculation and motion tracking via EPID. Strahlenther Onkol 185: 425-430, 2009.
35. Persson GF, Nygaard DE, Munck Af Rosenschöld P, Richter Vogelius I, Josipovic M, Specht L and Korreman SS: Artifacts in conventional computed tomography (CT) and free breathing four-dimensional $\mathrm{CT}$ induce uncertainty in gross tumor volume determination. Int J Radiat Oncol Biol Phys 80: 1573-1580, 2011

36. Bedi C, Kron T, Willis D, Hubbard P, Milner A and Chua B: Comparison of radiotherapy treatment plans for left-sided breast cancer patients based on three- and four-dimensional computed tomography imaging. Clin Oncol (R Coll Radiol) 23: 601-607, 2011.

37. Frazier RC, Vicini FA, Sharpe MB, Yan D, Fayad J, Baglan KL, Kestin LL, Remouchamps VM, Martinez AA and Wong JW: Impact of breathing motion on whole breast radiotherapy: A dosimetric analysis using active breathing control. Int J Radiat Oncol Biol Phys 58: 1041-1047, 2004.

38. Ding MS, Li JS, Deng J, Fourkal E and Ma CM: Dose correlation for thoracic motion in radiation therapy of breast cancer. Med Phys 30: 2520-2529, 2003.

39. Yue NJ, Li X, Beriwal S, Heron DE, Sontag MR and Huq MS: The intrafraction motion induced dosimetric impacts in breast 3D radiation treatment: A 4DCT based study. Med Phys 34: 2789-2800, 2007.

40. Luxton G, Hancock SL and Boyer AL: Dosimetry and radiobiologic model comparison of IMRT and 3D conformal radiotherapy in treatment of carcinoma of the prostate. Int J Radiat Oncol 59: 267-284, 2004

41. Fisher J,Scott C,Stevens R,Marconi B, Champion L, Freedman GM, Asrari F, Pilepich MV, Gagnon JD and Wong G: Randomized phase III study comparing Best Supportive Care to Biafine as a prophylactic agent for radiation-induced skin toxicity for women undergoing breast irradiation: Radiation therapy oncology group (RTOG) 97-13. Int J Radiat Oncol 48: 1307-1310, 2000.

42. Cai J, Read PW and Sheng K: The effect of respiratory motion variability and tumor size on the accuracy of average intensity projection from four-dimensional computed tomography: An investigation based on dynamic MRI. Med Phys 35: 4974-4981, 2008.

43. Park K, Huang L, Gagne H and Papiez L: Do Maximum Intensity Projection Images Truly Capture Tumor Motion? Int J Radiat Oncol Biol Phys 73: 618-625, 2009.

This work is licensed under a Creative Commons Attribution-NonCommercial-NoDerivatives 4.0 International (CC BY-NC-ND 4.0) License. 